\title{
Guanacos y ecología isotópica en el norte del Neuquén: El registro de Cueva Huenul 1
}

\author{
Guanacos and isotopic ecology in northern Neuquen: \\ The record from Cueva Huenul 1
}

\author{
Ramiro Barberena ${ }^{1 *}$, Augusto Tessone ${ }^{2}$, María Nella \\ Quiroga ${ }^{3}$, Florencia Gordón ${ }^{4}$, Carina Llano ${ }^{*}$, Alejandra \\ Gasco $^{6}$, Jimena Paiva y Andrew Ugan ${ }^{8}$
}

\begin{abstract}
${ }^{1 *}$ CONICET, Laboratorio de Paleoecología Humana, Facultad de Ciencias Exactas y Naturales, Universidad Nacional de Cuyo, Mendoza, Argentina. E-mail: ramidus28@gmail.com ${ }^{5}$ E-mail: Ilano.carina@gmail.com.6E-mail: soljandra@gmail.com

${ }^{2}$ CONICET, Instituto Nacional de Geocronología y Geología Isotópica, Buenos Aires, Argentina. CONICET Universidad Nacional de La Plata, La Plata, Buenos Aires, Argentina. E-mail: gutitessone@gmail.com. ${ }^{3}$ Facultad de Filosofía y Letras, Universidad Nacional de Cuyo, Mendoza, Argentina. E-mail: maru235@hotmail.com ${ }^{4}$ CONICET - Universidad Nacional de La Plata, Facultad de Ciencias Naturales y Museo, La Plata, Argentina. E-mail: fgordon2007@gmail.com.

${ }^{7}$ Laboratorio de Paleoecología Humana, Facultad de Ciencias Exactas y Naturales, Universidad Nacional de Cuyo, Mendoza, Argentina. E-mail: jimapaiva@gmail.com

${ }^{8}$ Far Western, Davis, California, Estados Unidos de Norteamérica. E-mail: Andrew.ugan@gmail.com
\end{abstract}

\begin{abstract}
Resumen
En este trabajo se presentan los primeros resultados de ecología isotópica regional para el extremo norte de la provincia del Neuquén (Argentina). Esta información es clave para la reconstrucción de cambios ecológicos y paleodietas humanas a través del tiempo. Se exponen los resultados obtenidos a partir de la medición de isótopos estables de carbono $\left({ }^{13} \mathrm{C} /{ }^{12} \mathrm{C}\right)$ y nitrógeno $\left({ }^{15} \mathrm{~N} /{ }^{14} \mathrm{~N}\right)$ sobre el colágeno óseo de 39 muestras de fauna y una muestra humana procedentes de sitios arqueológicos de la localidad Barrancas-Buta Ranquil. Se presentan datos para cinco especies silvestres, aunque el foco del análisis se centra en guanaco (Lama guanicoe). La información es interpretada en el marco de los resultados obtenidos de muestreos de vegetación en la región, los cuales indican la distribución y abundancia de especies con las distintas vías fotosintéticas. Debido a su posición, adyacente al ecotono entre las comunidades fitogeográficas del Monte y la Patagonia, la región ofrece un contexto adecuado para el análisis de cambios ecológicos a través del tiempo.

La totalidad de las especies vegetales relevadas en el muestreo sistemático siguen la vía fotosintética $C_{3}$. En concordancia con esto, los valores de especies animales fueron relacionados a una cadena trófica $\mathrm{C}_{3}$. El entierro humano del sitio Buta Có Abajo registró valores que sugieren una dieta compuesta por guanacos y que además incluyó animales pequeños con valores más enriquecidos. En cuanto a los guanacos, se registraron diferencias isotópicas entre las muestras asignadas al Holoceno temprano y el tardío; estas últimas reflejan un mayor consumo de especies con vía fotosintética $C_{3}$. Por último, en este conjunto de muestras, no se registró relación entre la altitud y los valores isotópicos de $\delta^{13} \mathrm{C} y \delta^{15} \mathrm{~N}$, mientras que se observaría una tendencia latitudinal en la que los camélidos del centro sur de Mendoza presentan un mayor consumo de especies vegetales $C_{4}$ que las procedentes del norte de Neuquén.
\end{abstract}

Palabras clave: Isótopos estables; Paleoecología de guanacos; Patagonia septentrional; Ecología del Monte; Paleodieta humana.

\section{Abstract}

In this work we present the first systematic results about regional isotopic ecology in northern Neuquén Province (Argentina). This information is essential to reconstruct ecological changes and human paleodiets through time. We conducted analyses of stable isotopes of carbon $\left({ }^{13} \mathrm{C} /{ }^{12} \mathrm{C}\right)$ and nitrogen $\left({ }^{15} \mathrm{~N} /{ }^{14} \mathrm{~N}\right)$ on the collagen fraction of bone from one human sample and 39 faunal samples from archaeological sites in Barrancas-Buta Ranquil locality. The results correspond to five wild species, though analysis is focused in guanaco (Lama guanicoe). The information produced is interpreted in the framework of regional botanical surveys that show the differential abundance of species with 
different photosynthetic pathways. Due to its position, next to the ecotone between the Monte and Patagonia phytogeographical provinces, the area is a sensitive spot to test ecological changes through time.

The species recorded in the botanical transects follow the $C_{3}$ photosynthetic pathway. Accordingly, the values produced for faunal samples reveal $\mathrm{C}_{3}$-based trophic chains. The human burial from Buta Có Abajo shows values indicative of a diet based on camelids, which would have also included small animals with higher isotope values. The samples analyzed for guanaco show differences between the early and late Holocene components from $\mathrm{CH} 1$, where the more recent assemblage shows a more intense consumption of $C_{3}$ plants. By integrating data available for neighbor southern Mendoza region, we evaluate regional isotopic patterns associated to altitude and latitude. We do not recorded strong relations between altitude and $\delta^{13} \mathrm{C}$ and $\delta^{15} \mathrm{~N}$ values. On the other hand, there is a weak latitudinal pattern in $\delta^{13} \mathrm{C}$ values, which show more depleted values in $\mathrm{CH} 1$ compared to settings located further north.

Keywords: Stable isotopes; Guanaco paleoecology; Northern Patagonia; Monte ecology; Human paleodiet.

El objetivo de este trabajo es presentar los primeros resultados sistemáticos de ecología isotópica regional para el extremo norte de la provincia del Neuquén (Argentina). Esta información es clave para la reconstrucción de cambios ecológicos y paleodietas humanas a través del tiempo. Se exponen los resultados obtenidos a partir de la medición de isótopos estables de carbono $\left({ }^{13} \mathrm{C} /{ }^{12} \mathrm{C}\right)$ y nitrógeno $\left({ }^{15} \mathrm{~N} /{ }^{14} \mathrm{~N}\right)$ en la fracción de colágeno óseo de 39 muestras de fauna y una muestra humana procedentes de sitios arqueológicos de la localidad BarrancasButa Ranquil. La región estudiada se emplaza en una posición adyacente al ecotono entre las comunidades fitogeográficas del Monte y la Patagonia (Movia et al. 1982), en una región de clima árido y semiárido asociado a la transición de los sistemas de circulación atmosférica Subtropical y Templado (Páez et al. 2004). Esta posición cercana al ecotono ofrece un contexto favorable para el análisis de cambios ecológicos a través del tiempo.

Se presentan datos para cinco especies silvestres, aunque el foco del análisis se centra en las muestras de camélidos procedentes del sitio Cueva Huenul 1 (Barberena 2015), que es el taxón más representado en el registro arqueológico regional (Barberena et al. 2015;

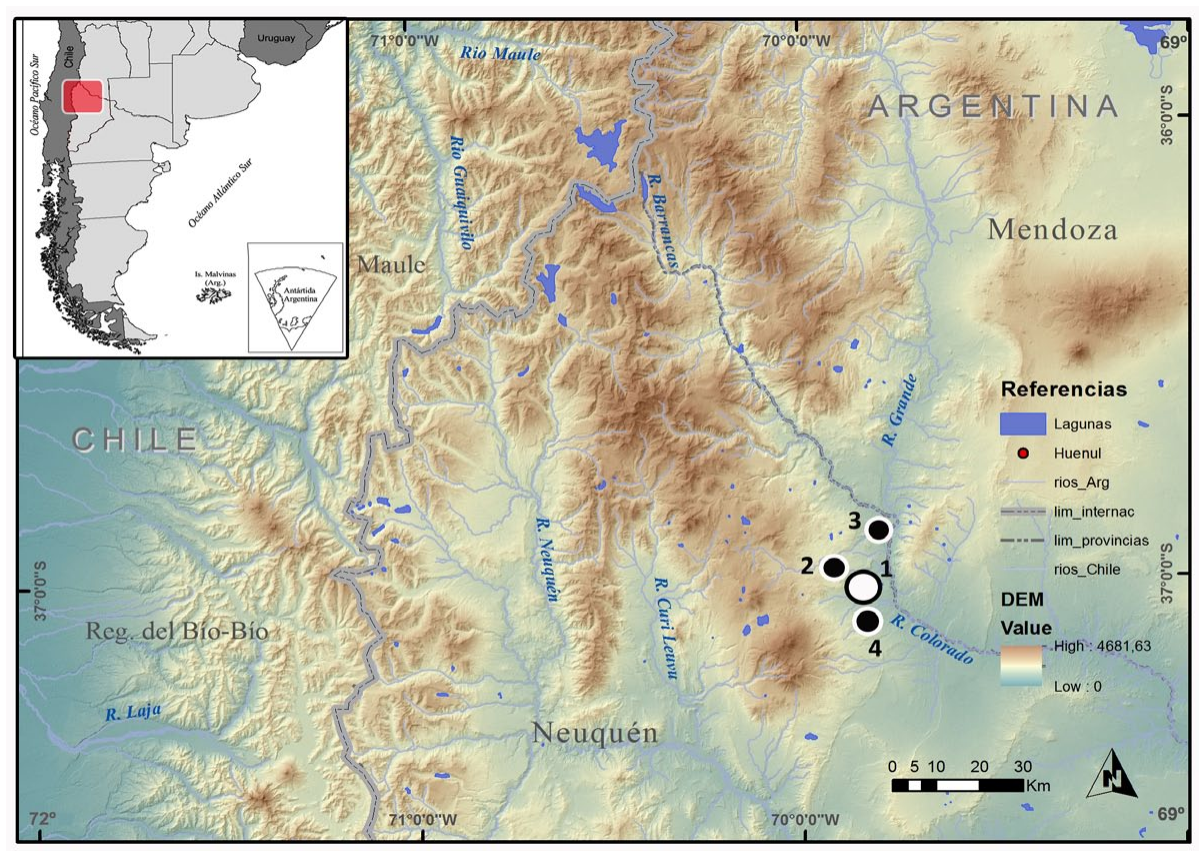

Neme et al. 2013; Rindel 2017). La información isotópica es interpretada en el marco de los resultados obtenidos de muestreos de vegetación en la región, los cuales indican la distribución y abundancia de especies con las distintas vías fotosintéticas. Estos datos producidos en escala de micro-región permiten complejizar patrones sobre la ecología isotópica de camélidos desarrollados en escalas espaciales más amplias (Barberena et al. 2009; Gil et al. 2016; Tessone et al. 2014), al tiempo que aportan información útil de referencia para interpretar paleodietas humanas en el norte neuquino.

\section{Materiales y métodos}

\section{Materiales}

La colección procede de cuatro sitios arqueológicos del norte de la provincia del Neuquén (Figura 1). Se analizó un total de 39 muestras con la siguiente procedencia: Cueva Huenul 1 (CH1), $n=35$ (36 $57^{\prime} \mathrm{S}, 69^{\circ} 49^{\prime} \mathrm{O}, 1000$ msnm); Cueva Yagui, $n=2\left(36^{\circ} 56^{\prime} S, 69^{\circ} 53^{\prime} \mathrm{O}, 1370 \mathrm{msnm}\right)$; Cueva Pava, $\mathrm{n}=1\left(36^{\circ} 0^{\prime} \mathrm{S}, 69^{\circ} 49^{\prime} \mathrm{O}, 1090 \mathrm{msnm}\right)$ y Entierro Buta Có Abajo, $n=1$ (36 $54^{\prime} \mathrm{S}, 69^{\circ} 47^{\prime} \mathrm{O}, 990$ msnm; Figura 1). De este conjunto, 11 muestras fueron recuperadas en superficie y 28 en estratigrafía. Una muestra es de Homo sapiens y 38 son de fauna: 30 fueron asignadas a Lama guanicoe, seis a Ctenomys sp., dos a Lagidium viscacia, una a Dasipodidae y una a Rhea sp.

El sitio $\mathrm{CH} 1$ provee la muestra principal para el análisis, lo que se debe al avanzado desarrollo

Figura 1. Ubicación de los sitios incluidos en este trabajo: 1) $\mathrm{CH}$, 2) Cueva Yagui, 3) Entierro Buta Có Abajo y 4) Cueva Pava.

Figure 1. Location of the sites studied in this paper: 1) $\mathrm{CH} 1,2)$ Cueva Yagui, 3) Entierro Buta Có Abajo and 4) Cueva Pava. 
de las excavaciones y a las excelentes condiciones de preservación de los depósitos estratificados. La secuencia sedimentaria de $\mathrm{CH} 1$ se extiende en forma discontinua durante los últimos 16.000 años calendáricos, ofreciendo un archivo para la evaluación de procesos temporales de cambio ecológico y arqueológico (Barberena 2015; Barberena et al. 2015).

\section{Métodos}

Para el muestreo de vegetación, que fue desarrollado en el entorno de $\mathrm{CH} 1$, se siguió el método de transectas tomando puntos de intercepción (Leis et al. 2015), el cual permite muestrear vegetación arbustiva y graminoide correspondiente con la fitogeografía del área de estudio. Se utilizó una cinta métrica, dos estacas y una aguja. La cinta se tensó paralela al suelo con dos estacas y la aguja se descendió verticalmente a la superficie del suelo a la distancia elegida en forma sistemática. Los puntos de intercepción se establecieron aleatoriamente utilizando una hoja de cálculo. Se tomaron 20 puntos por cada una de las 24 transectas, cuya longitud se estableció en 20 m (Mostacedo y Fredericksen 2000). Se registró la morfología de cada planta (p.e. hierba, graminoide, subarbusto, arbusto, trepadora, epífita).

Las muestras óseas fueron determinadas a nivel anatómico y taxonómico mediante el uso de colecciones de referencia. Para la selección de las muestras se tomaron en cuenta criterios anatómicos, etarios y cronológicos que minimizaran las posibilidades de muestrear un mismo individuo en forma redundante. Los valores isotópicos aquí presentados han sido obtenidos en dos laboratorios diferentes, cuyos métodos se describen a continuación. El conjunto mayor de muestras $(n=28)$ se analizó en el Instituto de Geocronología y Geología Isotópica (INGEIS, CONICET-UBA, Argentina). Se emplearon técnicas estándar para la extracción del colágeno óseo (Tykot 2004). Se efectuó la extracción con tejido cortical óseo que presentó buen aspecto visual. La limpieza mecánica fue realizada con un torno pequeño y posteriormente se enjuagó la muestra con agua bidestilada en un baño de ultrasonido. El proceso de desmineralización ósea se realizó con $\mathrm{HCl} 2 \%$ por 72 horas. Antes y después del mismo se dispuso el material por 24 horas en $\mathrm{NaOH}$ $0,1 \mathrm{M}$ con el fin de eliminar los compuestos orgánicos post-depositacionales.

Para estimar el rendimiento del colágeno la muestra fue pesada previo al ataque con reactivos y con posterioridad a la extracción. Dicho rendimiento y la relación carbono/ nitrógeno (C/N) son los parámetros que se utilizan para el control diagenético de las muestras (Ambrose 1990). La medición de las relaciones isotópicas en la fracción colagénica ósea se realizó con un analizador elemental Carlo Erba (CHONS) conectado a través de una interfaz ThermoConflo IV a un espectrómetro de masas de relaciones isotópicas de flujo continuo ThermoDelta $\mathrm{V}$. Un segundo conjunto de muestras $(n=12)$ fue preparado en el Museo de Historia Natural de San Rafael (Mendoza, Argentina) y luego procesado en la University of Wyoming
Tabla 1. Resultados del relevamiento botánico en la localidad Huenul.

Table 1. Results of the botanical survey in Huenul locality.

\begin{tabular}{lrr}
\hline Especie & Valor absoluto & Valor relativo \\
\hline Acantholippia seriphioides & 2 & 1,39 \\
Chuquiraga oppositifolia & 12 & 8,33 \\
Glandularia crithmifolia & 2 & 1,39 \\
Larrea divaricata & 49 & 34,03 \\
Monttea aphylla & 19 & 13,19 \\
Poaceae aff. Pappostipa chrysophylla & 10 & 6,94 \\
Poaceae & 13 & 9,03 \\
Prosopis alpataco & 3 & 2,08 \\
Schinus sp. & 33 & 22,92 \\
\hline INDET & 1 & 0,69 \\
TOTAL & 144 & 100 \\
\hline
\end{tabular}

(Estados Unidos). En este caso, las muestras fueron desmineralizadas en $\mathrm{HCl} 0,6 \mathrm{~N}$ a $4^{\circ} \mathrm{C}$ y tratadas con $\mathrm{KOH}$ $5 \%$ para remover contaminantes orgánicos. Luego se las sumergió en una mezcla 2:1:0,8 de metanol, cloroformo y agua durante 24 horas. Fueron liofilizadas y enjuagadas en $\mathrm{KOH} 5 \%$. Luego, se pesaron para obtener el rendimiento del colágeno. Aproximadamente 100 mg de colágeno liofilizado se gelatinizó en $5 \mathrm{ml}$ de agua acidificada ( $\mathrm{pH} 3$ ) durante 24 horas a $120^{\circ} \mathrm{C}$. Las fases solubles e insolubles del agua fueron separadas por filtración y la fase soluble fue liofilizada. Se midieron los valores de $\delta^{13} \mathrm{C}$ y $\delta^{15} \mathrm{~N}$ con un Analizador Elemental Costech 4010 conectado a Thermo Delta Plus XP IRMS. La desviación estándar obtenida para los valores de $\delta^{13} \mathrm{C}$ y $\delta^{15} \mathrm{~N}$ fue de $\pm 0,3 \%$. Los valores de la relación $\mathrm{C} / \mathrm{N}$ considerados normales para huesos bien preservados se ubican entre 2,9 y 3,6 (DeNiro 1985).

\section{Resultados}

Relevamiento fitogeográfico en la localidad Huenul La totalidad de las especies relevadas en el muestreo sistemático siguen la vía fotosintética $C_{3}$ (Tabla 1 ). Predominan los taxones arbustivos Larrea divaricata (jarilla) y Schinus sp. (molle), que dan cuenta de un 57\% de los individuos registrados. Aunque no fueron observadas en el muestreo sistemático, cabe señalar la presencia de taxones $C_{4}$ en baja frecuencia, incluyendo Aristida subulata, Euphorbia portulacoides y Tillandsia bryoides.

\section{Ecología isotópica}

En la Tabla 2 se presentan los resultados isotópicos en conjunto con la información contextual a nivel taxonómico, espacial y temporal. A su vez, se consignan las muestras datadas en forma directa mediante fechados ${ }^{14} \mathrm{C}$ AMS en la University of Arizona, incluyendo los valores de $\delta^{13} \mathrm{C}$ medidos en asociación con cada fechado.

Del total de las muestras analizadas, 37 presentaron 
Tabla 2. Resultados isotópicos para el norte de Neuquén.

Table 2. Isotopic results for northern Neuquén.

\begin{tabular}{|c|c|c|c|c|c|c|c|c|c|c|c|c|c|c|}
\hline $\mathrm{N}$ & Sitio & Unidad & Nivel & Taxon & Elemento & Edad & Desvío & $\delta^{13} \mathrm{C}$ fecha AMS & Codigo & Amt \%N & Amt $\% \mathrm{C}$ & $\mathrm{C} / \mathrm{N}$ & $\delta^{13} \mathrm{C}$ & $\delta^{15} \mathrm{~N}$ \\
\hline 1 & Buta Co Abajo & entierro & & Homo sapiens & costilla & & & & AlE 30938 & & & 3,3 & $-17,5$ & 11 \\
\hline 2 & $\mathrm{CH} 1$ & A 1 & $1 \mathrm{~W}(10-20 \mathrm{~cm})$ & Lama guanicoe & metapodio (diáfisis) & 373 & 43 & $-20,4$ & AIE 32271 & 13,5 & 35,8 & 3,1 & $-19,6$ & 5,4 \\
\hline 3 & $\mathrm{CH} 1$ & A 1 & $3 \mathrm{E}(30-40 \mathrm{~cm})$ & Lama guanicoe & tibia (diáfisis) & 1590 & 46 & $-19,9$ & AlE 32272 & 15,1 & 40 & 3,1 & $-18,9$ & 5,9 \\
\hline 4 & $\mathrm{CH} 1$ & A 1 & $4 \mathrm{E}(40-50 \mathrm{~cm})$ & Lama guanicoe & húmero (diáfisis) & 9295 & 90 & $-17,7$ & AIE 32273 & 14,4 & 38,6 & 3,1 & $-17,4$ & 5,6 \\
\hline 5 & $\mathrm{CH} 1$ & A 1 & $4 \mathrm{~W}(40-50 \mathrm{~cm})$ & Lama guanicoe & radio cúbito (diáfisis) & 9375 & 91 & $-18,1$ & AIE 32274 & 14,9 & 40,1 & 3,1 & $-17,4$ & 4,4 \\
\hline 6 & $\mathrm{CH} 1$ & B 1 & 2 & Lama guanicoe & húmero (diáfisis) & 1269 & 46 & $-20,2$ & AlE 32275 & 15,1 & 40,4 & 3,1 & $-19,7$ & 5,6 \\
\hline 7 & $\mathrm{CH} 1$ & B 1 & 6 & Lama guanicoe & radio cúbito (diáfisis) & 1753 & 47 & $-20,2$ & AIE 32276 & 14,7 & 39 & 3,1 & -19 & 4,9 \\
\hline 8 & C. Pava * & F 9 & $8(250-260 \mathrm{~cm})$ & Lama guanicoe & radio ulna (diáfisis) & & & & AIE 32593 & 11,5 & 42,8 & 4,3 & --- & --- \\
\hline 9 & $\mathrm{CH} 1$ & B 1 & 5 & Lama guanicoe & metatarso (proximal) & & & & AlE 32277 & 15,3 & 40,2 & 3,1 & $-19,1$ & 5,3 \\
\hline 10 & $\mathrm{CH} 1$ & superficie & & Lama guanicoe & metatarso (proximal) & & & & AIE 32278 & 14,8 & 39,1 & 3,1 & $-19,5$ & 4,4 \\
\hline 11 & $\mathrm{CH} 1$ & B 1 & $6(70-80 \mathrm{~cm})$ & Lama guanicoe & metatarso (proximal) & & & & AIE 32279 & 16,8 & 45,7 & 3,2 & $-19,6$ & 4,8 \\
\hline 12 & $\mathrm{CH} 1$ & superficie & & Lama guanicoe & metapodio (diáfisis) & & & & AIE 32281 & 16,9 & 45,9 & 3,2 & $-19,5$ & 5 \\
\hline 13 & $\mathrm{CH} 1$ & A 1 & superficie & Lama guanicoe & metapodio (distal) & & & & AlE 32282 & 15,9 & 45,6 & 3,3 & $-19,9$ & 6,3 \\
\hline 14 & $\mathrm{CH} 1$ & A 1 & $3 E$ & Lama guanicoe & pelvis & & & & AIE 32283 & 16,7 & 49,4 & 3,4 & $-20,3$ & 6 \\
\hline 15 & $\mathrm{CH} 1$ & B 1 & 3 & Lama guanicoe & calcáneo & & & & AlE 32768 & 16,2 & 46,4 & 3,3 & $-19,3$ & 5,7 \\
\hline 16 & $\mathrm{CH} 1$ & B 1 & 5 & Lama guanicoe & falange $2 \mathrm{da}$ & & & & AlE 32284 & 16,6 & 45,3 & 3,2 & $-19,9$ & 4,8 \\
\hline 17 & $\mathrm{CH} 1$ & A 1 & $2 W$ & Lama guanicoe & hemimandibula & & & & AIE 32285 & 15,3 & 42 & 3,2 & $-19,3$ & 5,5 \\
\hline 18 & $\mathrm{CH} 1$ & superficie & & Lama guanicoe & metacarpo (proximal) & & & & AIE 32288 & 16,4 & 47,7 & 3,4 & $-20,2$ & 4,7 \\
\hline 19 & Cueva Yagui & superficie & & Lama guanicoe & calcáneo & & & & AIE 32769 & 16,4 & 45,2 & 3,2 & $-20,1$ & 6 \\
\hline 20 & Cueva Yagui & superficie & & Lama guanicoe & falange 1ra delantera & & & & AIE 32289 & 17,2 & 47,2 & 3,2 & $-19,3$ & 5,2 \\
\hline 21 & $\mathrm{CH} 1$ * & superficie & & Lama guanicoe & falange $2 \mathrm{da}$ & & & & AIE 32290 & 15 & 47,8 & 3,7 & --- & --- \\
\hline 22 & $\mathrm{CH} 1$ & superficie & & Lama guanicoe & metapodio (distal) & & & & AIE 32291 & 17,1 & 47,8 & 3,3 & $-20,4$ & 5,2 \\
\hline 23 & $\mathrm{CH} 1$ & superficie & & Lama guanicoe & metapodio (distal) & & & & AlE 32292 & 16 & 44,1 & 3,2 & $-19,4$ & 5,4 \\
\hline 24 & $\mathrm{CH} 1$ & superficie & & Lama guanicoe & metapodio (distal) & & & & AIE 32293 & 16,2 & 47,1 & 3,4 & -21 & 5,8 \\
\hline 25 & $\mathrm{CH} 1$ & A1 & 2 & Lama guanicoe & radioulna & & & & A 233 & 15,4 & 42,5 & 3,2 & -18 & 6,5 \\
\hline 26 & $\mathrm{CH} 1$ & $\mathrm{~A} 1$ & 3 & Lama guanicoe & vértebra lumbar & & & & A 236 & 14,6 & 41,7 & 3,3 & $-19,9$ & 5,3 \\
\hline 27 & $\mathrm{CH} 1$ & $\mathrm{~A} 1$ & 3 & Lama guanicoe & falange 1ra & & & & A 237 & 15,2 & 42,9 & 3,3 & $-19,9$ & 4,2 \\
\hline 28 & $\mathrm{CH} 1$ & $\mathrm{~A} 1$ & 4 & Lama guanicoe & tibia & & & & A 239 & 15 & 41,6 & 3,2 & $-19,5$ & 4,5 \\
\hline 29 & $\mathrm{CH} 1$ & $\mathrm{~A} 1$ & 4 & Lama guanicoe & radioulna & & & & A 241 & 14,8 & 41 & 3,2 & $-18,2$ & 4,9 \\
\hline 30 & $\mathrm{CH} 1$ & B1 & 5 & Lama guanicoe & falange 1ra & & & & A 244 & 15,1 & 41,6 & 3,2 & $-19,8$ & 5,1 \\
\hline 31 & $\mathrm{CH} 1$ & superficie & & Lama guanicoe & falange 1ra delantera & & & & AlE 32286 & 17,1 & 48,6 & 3,3 & $-20,3$ & 5,5 \\
\hline 32 & $\mathrm{CH} 1$ & B 1 & 6 & Dasipodidae & placa & & & & AIE 32594 & 15,4 & 45,8 & 3,5 & $-15,5$ & 9,2 \\
\hline 33 & $\mathrm{CH} 1$ & A 1 & $7 W$ & Lagidium viscacia & metapodio & & & & AlE 32287 & 14,5 & 41,9 & 3,4 & $-19,6$ & 5,9 \\
\hline 34 & $\mathrm{CH} 1$ & A1 & 2 & Ctenomys sp. & hemimandibula & & & & A 234 & 14 & 40,5 & 3,3 & $-20,3$ & 3,3 \\
\hline 35 & $\mathrm{CH} 1$ & $\mathrm{~A} 1$ & 4 & Ctenomys sp. & hemimandibula & & & & A 235 & 15,4 & 43,7 & 3,3 & $-18,7$ & 4,2 \\
\hline 36 & $\mathrm{CH} 1$ & $\mathrm{~A} 1$ & 5 & Ctenomys sp. & hemimandibula & & & & A 238 & 15,2 & 42,8 & 3,2 & $-19,9$ & 5,3 \\
\hline 37 & $\mathrm{CH} 1$ & B1 & 5 & Ctenomys sp. & hemimandibula & & & & A 245 & 14,7 & 41,1 & 3,2 & $-19,8$ & 3,2 \\
\hline 38 & $\mathrm{CH} 1$ & B1 & 6 & Ctenomys sp. & hemimandibula & & & & A 246 & 15,3 & 43,5 & 3,3 & $-19,8$ & 3,5 \\
\hline 39 & $\mathrm{CH} 1$ & B1 & 1 & Rhea sp. & diáfisis & & & & A 243 & 15,2 & 42,2 & 3,2 & -21 & 6,6 \\
\hline
\end{tabular}

una relación C/N considerada adecuada (DeNiro 1985), con una media de 3,2 $\pm 0,1$. Dos muestras de guanaco registran una relación $\mathrm{C} / \mathrm{N}$ por fuera del rango que indica preservación adecuada del colágeno y son excluidas del análisis (Tabla 2).

Los valores para guanaco tienen un promedio de $-19,5$ $\pm 0,8 \%$ para $\delta^{13} \mathrm{C}$ y $5,3 \pm 0,6 \%$ o para $\delta^{15} \mathrm{~N}(\mathrm{~N}=29, \mathrm{r}$ $=-0,07 ;$ p 0,71), mientras que las muestras de Ctenomys sp. tienen una media de $-19,7 \pm 0,6 \%$ o para $\delta^{13} \mathrm{C}$ y $3,9 \pm$ $0,9 \%$ para $\delta^{15} \mathrm{~N}(\mathrm{~N}=5)$. Por último, la muestra de Lagidium viscacia tiene valores de $-19,6$ para $\delta^{13} \mathrm{C}$ y 5,9 para $\delta^{15} \mathrm{~N}$, que resultan indistinguibles del conjunto mayor de Lama guanicoe. El valor de $\delta^{13} \mathrm{C}$ disponible para Rhea sp. es -21 y el de $\delta^{15} \mathrm{~N}$ es 6,6 . La muestra correspondiente a Dasipodidae presenta valores de $\delta^{13} \mathrm{C}$ de $-15,5$ y de $\delta^{15} \mathrm{~N}$ de 9,2 , datos que permiten diferenciarla de las demás.

El entierro humano del sitio Buta Có Abajo: contexto e isótopos

En el marco de este proyecto se ha recuperado un único entierro humano, procedente del sitio Buta Có
Abajo. En este sitio se registraron restos pertenecientes a un individuo colocados en una pequeña oquedad producto de erosión de un afloramiento de ignimbritas correspondientes a la Fm. Tilhué.

Este individuo está representado por cráneo, cúbito izquierdo y fragmentos de vértebras indeterminados. El cráneo se halla fragmentado y exhibe evidencias avanzadas de meteorización -estadios 3 y 4- (Behrensmeyer 1978). Presenta marcas de raíces y evidencias de pérdida ósea de ciertos elementos como la mandíbula y el maxilar superior izquierdo. Si bien el cráneo no parece estar modificado artificialmente, el estado de conservación no permite realizar una determinación precisa. En relación con la edad estimada, a partir de la secuencia de erupción dentaria podría tratarse de un individuo de entre 12 y 15 años (presenta M1 y M2 superiores derechos), aunque no se registraron las demás piezas dentarias. No obstante, la sutura esfenobasilar permanece aún sin fusionar, con lo cual sería un individuo menor a los 18 años. Por el carácter incompleto y fragmentario del material, la estimación del sexo resulta poco confiable. 


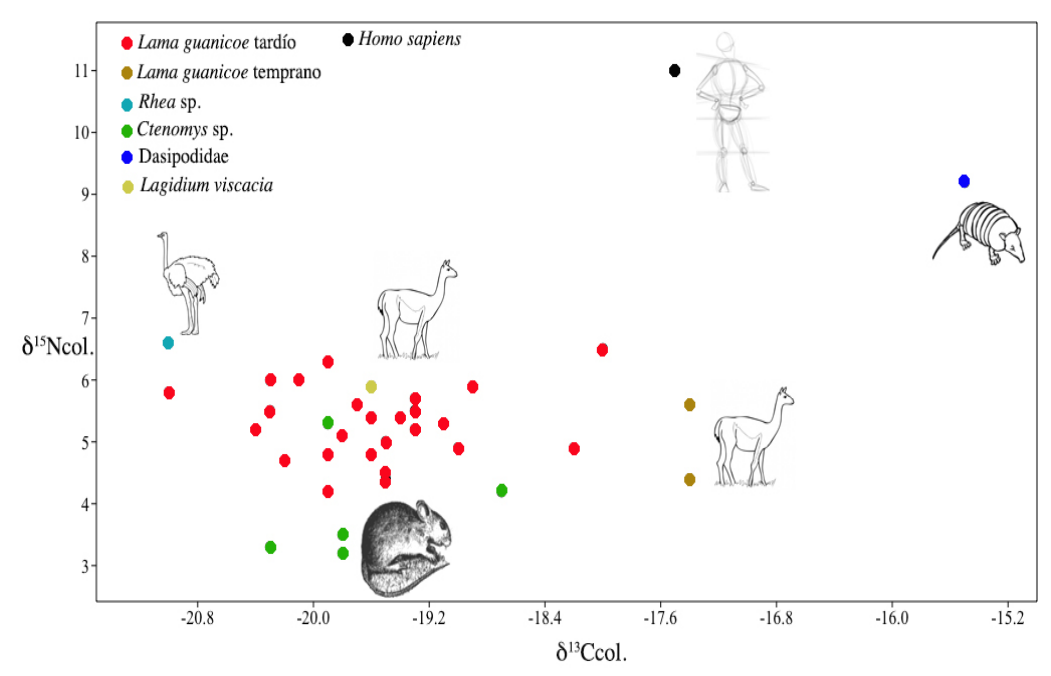

Figura 2. Relación entre los valores $d e d^{13} \mathrm{C}$ y $d^{15} \mathrm{~N}$ de las especies faunísticas.

Figure 2. Scatterplot of the $d^{13} \mathrm{C}$ y $d^{15} \mathrm{~N}$ values for faunal samples.

obtenidos recientemente en el noroeste de Neuquén y sur de Mendoza (Fernández et al. 2016; Gil et al. 2016; Gordón et al. 2017).

Sobre la base de estos datos puede realizarse una interpretación dietaria de la muestra humana del entierro de Buta Có Abajo, que presenta valores de $\delta^{13} \mathrm{Ccol} .=-17,5 \%$ y $\delta^{15} \mathrm{~N}=$ $11 \%$. Tomando en cuenta el amplio conjunto de determinaciones realizadas para guanacos de la región, se observa que los valores de $\delta^{13} \mathrm{C}$ y $\delta^{15} \mathrm{~N}$ sugieren una dieta que, además de los camélidos, incluyó recursos con valores más enriquecidos, como alguno de los animales pequeños muestreados en este trabajo -Dasipodidae-. Esto resulta interesante, ya que el estudio tafonómico y zooarqueológico del sitio $\mathrm{CH} 1$ indica que Lama guanicoe es el taxón más abundante vinculado a la conducta humana, que los restos correspondientes a pequeños roedores tienen una depositación principalmente asociada a aves rapaces (Fernández et al. 2012), y que los animales pequeños consumidos por los humanos se depositaron en muy bajas proporciones (Barberena et al. 2015). Esta interpretación se ve reforzada al comparar los valores con los obtenidos recientemente en una muestra del noroeste de Neuquén (Gordón et al. 2017). En promedio, estos individuos presentaron valores más bajos $\left(\delta^{13} \mathrm{C}=-18,3 ; \delta^{15} \mathrm{~N}=8,9\right)$. En este caso, el análisis a través de modelos de mezcla bayesianos permitió estimar proporciones elevadas de consumo de grandes animales (representando alrededor del $84 \%$ del total de la dieta), tales como Lama guanicoe y Rhea sp. Cuando se disponga de una muestra humana mayor y de determinaciones isotópicas sobre recursos vegetales se procederá a la utilización de este tipo de modelos para estimar en forma cuantitativa el aporte de las distintas clases de recursos (Gordón et al. 2017).

Lama guanicoe: vegetación y exploración de tendencias temporales

Para el análisis temporal, del total de muestras de guanaco analizadas se utilizan sólo aquellas seis que han sido datadas por AMS en forma directa (Tabla 2), lo cual provee un control de la posible existencia de migración vertical de materiales en la secuencia. Estas muestras representan los dos principales componentes arqueológicos definidos para $\mathrm{CH} 1$ : el componente 2 del Holoceno temprano (11000-10200 años cal AP), representado por dos muestras, y el componente 4 del Holoceno tardío (1500-300 años cal AP), representado por cuatro muestras (Barberena et al. 2015).

Con respecto a las dos muestras del Holoceno temprano, cabe señalar que no se puede descartar que procedan 
de un mismo individuo. Dado el pequeño tamaño de la muestra de este componente, no se puede definir un MNI mayor a 1. Por este motivo, las tratamos como una única muestra siguiendo un criterio conservador. Resulta interesante el valor de $\delta^{13} \mathrm{C}$ de $-17,4 \%$ o -que tienen ambas muestras-, que la distingue no sólo de las cuatro muestras tardías fechadas, sino de todo el conjunto de guanaco procedente del componente temporal tardío con una media de $-19,6 \pm 0,6$ y valores máximos y mínimos de -18 y $-20,4 \%$ o $(\mathrm{N}=26)$. Por el contrario, no se observan diferencias temporales en los valores de $\delta^{15} \mathrm{~N}$.

Más allá del carácter limitado del conjunto temprano, se registraría el consumo de especies vegetales con valores más enriquecidos que durante el Holoceno tardío. Un cálculo del posible aporte dietario de plantas $C_{4}$ sugiere una proporción de $22 \%$, que es mayor a lo estimado para las muestras tardías con aportes que varían entre 14 y $0 \%$. Aunque se requieren determinaciones locales, la diferencia puede reflejar una mayor abundancia de gramíneas $C_{4}$ en el Holoceno temprano. Dada la mayor tolerancia de estas plantas a condiciones de estrés hídrico y temperaturas elevadas (Ehleringer y Cerling 2001), esto puede deberse a condiciones de mayor aridez.
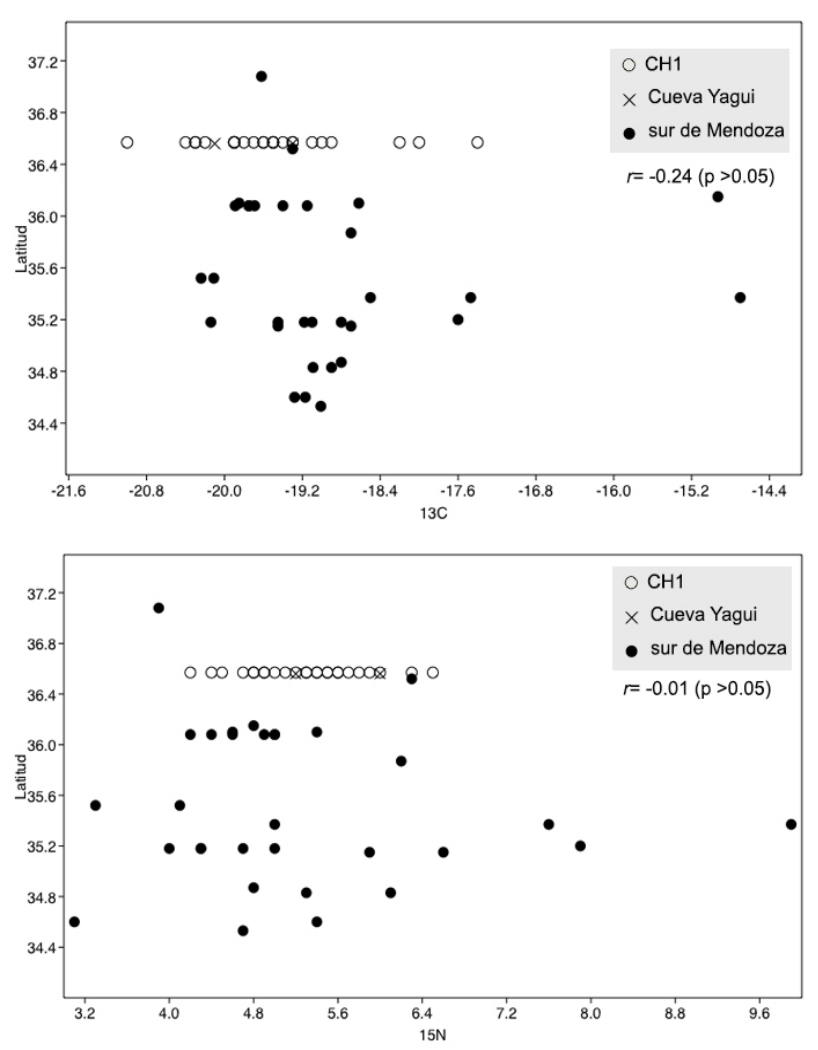

Figura 3. Relación entre latitud y valores de $\delta^{13} \mathrm{C}$ y $\delta^{15} \mathrm{~N}$ en muestras de Lama guanicoe del norte de Neuquén y sur de Mendoza.

Figure 3. Scatterplot between latitude and $\delta^{13} \mathrm{C}$ and $\delta^{15} \mathrm{~N}$ for Lama guanicoe samples from northern Neuquén and southern Mendoza.
Sin embargo, otra explicación posible es la existencia de condiciones de mayor humedad entre $14.800 \mathrm{y}$ 8.900 años cal AP, sugeridas por el registro polínico de Mallín Vaca Lauquen ( $36^{\circ} \mathrm{S}$ y $71^{\circ} \mathrm{O}$; Markgraf et al. 2008), que pudo haber producido un aumento relativo en la presencia de gramíneas -algunas de ellas $\mathrm{C}_{4}$ - en detrimento de arbustos.

Estos datos permiten comenzar a construir una ecología isotópica local para el norte de Neuquén, revelando que, por su similitud isotópica, algunos taxa no podrían ser discriminados. Este es el caso de Lama guanicoe y Lagidium viscacia. Por otra parte, y aunque los tamaños de muestra son muy pequeños, existe el potencial de discriminar el consumo de guanacos con respecto a Rhea sp. sobre la base de los valores de $\delta^{15} \mathrm{~N}$ y, con más claridad aún, con respecto a Dasipodidae sobre la base de ambos elementos químicos.

Lama guanicoe: tendencias espaciales

Contextualizamos los datos de camélidos de $\mathrm{CH} 1$ en el marco de los datos publicados por Gil et al. (2016) para el sur de Mendoza, incluyendo sólo los datos que proceden de contextos ubicados por debajo de $34^{\circ}$ de
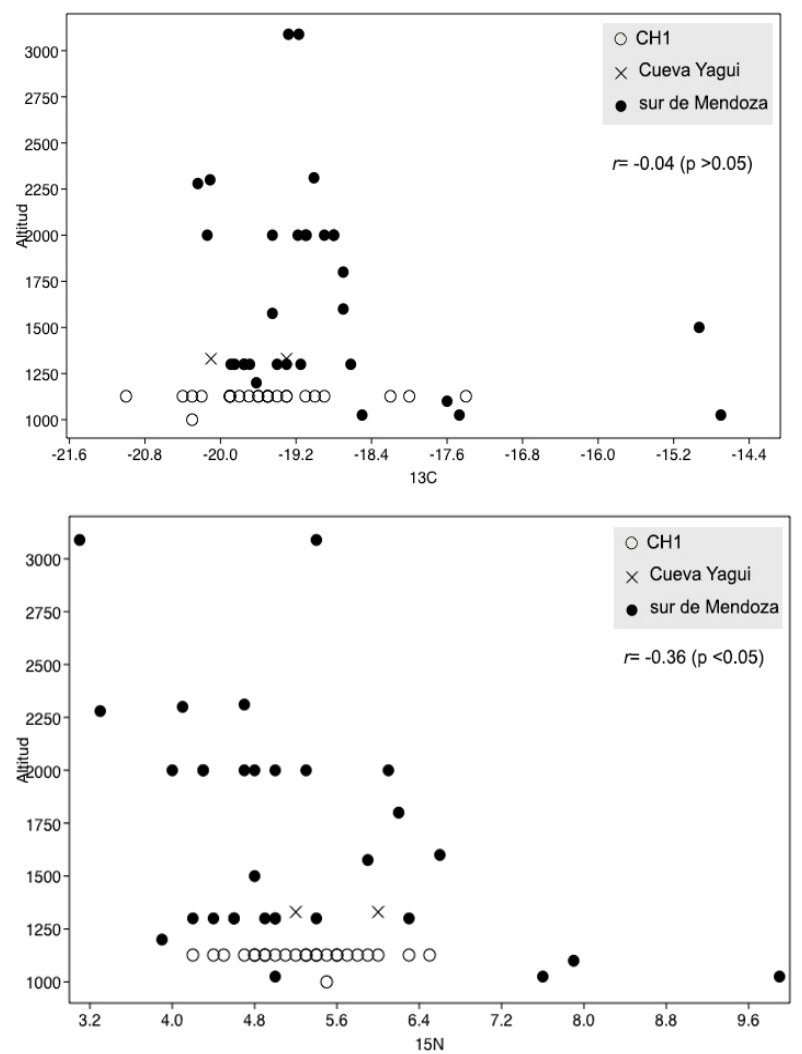

Figura 4. Relación entre altitud y valores de $\delta^{13} \mathrm{C}$ y $\delta^{15} \mathrm{~N}$ en muestras de Lama guanicoe del norte de Neuquén y sur de Mendoza.

Figure 4. Scatterplot between altitude and $\delta^{13} \mathrm{C}$ and $\delta^{15} \mathrm{~N}$ for Lama guanicoe samples from northern Neuquén and southern Mendoza. 
latitud sur $(\mathrm{N}=31)$, a fin de excluir contextos del norte de Mendoza y San Juan que resultan menos comparables a nivel ecológico.

En la Figura 3 se presentan las relaciones entre $\delta^{13} \mathrm{C}$ y $\delta^{15} \mathrm{~N}$ con la latitud y en la Figura 4 en relación con la altitud.

La relación entre la latitud y los valores de $\delta^{13} \mathrm{C}$ es débil, negativa y no significativa $(r=-0,24, p>0,05)$, mientras que con $\delta^{15} \mathrm{~N}$ es nula y no significativa $(r=-0,01 ; p$ $>0,05)$. Las tendencias en $\delta^{13} \mathrm{C}$ pueden marcar que los guanacos del norte de Neuquén (36 $57^{\prime}$ ) presentan en promedio un menor consumo de gramíneas $C_{4}$ que las muestras ubicadas a latitudes menores en el centro sur de Mendoza.

La relación entre la altitud y los valores isotópicos de $\delta^{13} \mathrm{C}$ es nula y no significativa $(r=-0,04, p>0,05)$, mientras que con $\delta^{15} \mathrm{~N}$ es negativa y estadísticamente significativa ( $r=$ $-0,36 ; p<0,05)$. Análisis previos dirigidos a evaluar este tema en escalas más amplias tampoco verificaron una correlación fuerte entre la altitud y los valores de $\delta^{13} \mathrm{C}$ o entre la altitud y los valores de $\delta^{15} \mathrm{~N}$ (Barberena et al. 2009; Gil et al. 2016). Aunque cabe esperar diferencias en la composición de las comunidades vegetales entre distintos pisos altitudinales (Cavagnaro 1988; Llano 2009), los resultados disponibles para la franja ubicada entre $34^{\circ} \mathrm{y}$ $37^{\circ}$ sur no marcan diferencias significativas. En el caso de los datos de $\delta^{13} \mathrm{C}$, y en forma consistente con lo registrado en el muestreo vegetacional en la localidad Huenul, esto puede deberse a la baja frecuencia de plantas $C_{4}$ aún en contextos de Monte y a baja altitud.

\section{Perspectivas}

Sobre la base del relevamiento fitogeográfico y los datos isotópicos aquí presentados, se ha comenzado a construir una ecología isotópica para el ecosistema de Monte que domina en el entorno de la localidad arqueológica Huenul. Sumado a información que se ha producido recientemente para localidades cercanas de Neuquén y Mendoza (Fernández et al. 2016; Gil et al. 2016; Gordón et al. 2017), estos datos proveen una base sólida para la reconstrucción paleodietaria humana. Este marco será clave para evaluar la amplitud dietaria a través del tiempo a nivel de las proteínas animales, evaluadas en función de marcadores isotópicos en colágeno, y de la dieta total. Este debate es significativo no sólo a nivel de la subsistencia humana, sino que tiene implicancias en términos de la organización espacial (Neme et al. 2013; Tessone et al. 2014). Con respecto al caso de guanaco, taxón para el que se ha presentado la mayor parte de la información, se observa que en la escala regional aquí discutida no hay tendencias marcadas en los valores isotópicos en relación con la altitud y la latitud. Más allá de la marcada heterogeneidad de paisaje en distancias pequeñas que caracteriza al norte de Neuquén, puede plantearse que todos los ecosistemas tendrían una señal de base dominante de tipo $\mathrm{C}_{3}$. A futuro, se debe avanzar en la instancia clave de producir valores locales para taxones vegetales, incluyendo aquellos que sabemos fueron consumidos por los humanos localmente (Llano y Barberena 2013), como aquellos que habrían sido consumidos por los herbívoros y omnívoros representados en el registro zooarqueológico.

Mendoza, agosto de 2017

\section{Agradecimientos}

Esta publicación es producto de los proyectos PICT 20121976, 2014-0940 y 201-0062 (Agencia Nacional de Promoción de la Ciencia y la Tecnología, Argentina). La Lic. Paula Sosa colaboró en la realización de los muestreos de vegetación. Se agradece a las autoridades de Barrancas (provincia del Neuquén), que facilitan enormemente nuestro trabajo, particularmente a Juvenal Urrutia, Paulina Valenzuela y Javier Siegel. A Estela Ducós y Nazareno Piperissa del INGEIS por la ayuda brindada en el análisis de las muestras. A Claudia Della Negra, Pablo Azar, Liliana Martínez y Estela M. Cúneo, de la Subsecretaría de Cultura de la Provincia del Neuquén, por toda la ayuda brindada. Finalmente, se agradecen las constructivas evaluaciones recibidas y se destaca la ayuda y paciencia de los editores de la Revista.

\section{Referencias citadas}

Ambrose, S.H. 1990. Preparation and characterization of bone and tooth collagen for stable carbon and nitrogen isotope analysis. Journal of Archaeological Science 17(4): 431-451.

Barberena, R. 2015. Cueva Huenul 1 Archaeological Site (Northwestern Patagonia, Argentina): Initial Colonization and mid-Holocene Demographic Retraction. Latin American Antiquity 26: 304-318.

Barberena, R., K. Borrazzo, A. Rughini, G. Romero, M.P. Pompei, C. Llano, M.E. de Porras, V. Durán, C.R. Stern, A. Re, D. Estrella, A. Forasiepi, F.J. Fernández, M. Chidiak, L. Acuña, A. Gasco \& M.N. Quiroga. 2015. Perspectivas arqueológicas para Patagonia septentrional: sitio Cueva Huenul 1 (Provincia del Neuquén, Argentina). Magallania 43 (1): 1-27.

Barberena, R., A.F. Zangrando, A.F. Gil, G.A. Martínez, G.G. Politis, L.A. Borrero \& G.A. Neme. 2009. Guanaco (Lama guanicoe) isotopic ecology in southern South America: spatial and temporal tendencies, and archaeological implications. Journal of Archaeological Science 36 (12): 2666-2675.

Behrensmeyer, A.K. 1978. Taphonomic and ecologic information from bone weathering. Paleobiology 4: 150-162.

Cavagnaro, J.B. 1988. Distribution of $C_{3}$ and $C_{4}$ grasses at different altitudes in a temperate arid region of Argentina. Oecologia 76 (2): 273-277.

DeNiro, M.J. 1985. Postmortem preservation and alteration of in vivo bone collagen isotope ratios in relation to paleodietary reconstruction. Nature 317: 806-809. 
Dawson, T.E., S. Mambelli, A.H. Plamboeck, P.H. Templer, K.P. Tu. 2002. Stable isotopes in plant ecology. Annual Review in Ecology and Systematics 33: 507-559.

Ehleringer, J.E. \& T.E. Cerling. 2001. Photosynthetic Pathways and Climate, in E.-D. Schulze, M. Heimann, S. Harrison, E. Holland, J. Lloyd, I.C. Prentice \& D.S. Schimel (eds.) Global Biogeochemical Cycles in the Climate System: 267-77. New York: Academic Press.

Fernández, F., A. Gil, A. Ugan \& G. Neme. 2016. Ecological conditions and isotopic diet $\left({ }^{13} \mathrm{C}\right.$ and $\left.{ }^{15} \mathrm{~N}\right)$ of Holocene caviomorph rodents in northern Patagonia. Journal of Arid Environments 127: 44-52.

Fernández, F.J., P. Teta, R. Barberena \& U.F.J. Pardiñas. 2012. Small mammal remains from Cueva Huenul 1, northern Patagonia, Argentina. Taphonomy and paleoenvironments since the Late Pleistocene. Quaternary International 278: 22-31.

Gil, A., A. Ugan, C. Otaola, G. Neme, M. Giardina \& L. Menéndez. 2016. Variation in camelid $\delta^{13} \mathrm{C}$ and $\delta^{15} \mathrm{~N}$ values in relation to geography and climate: Holocene patterns and archaeological implications in central western Argentina. Journal of Archaeological Science 66: 7-20.

Gordón, F., S.I. Perez, A. Hajduk, M. Lezcano \& V. Bernal. 2017. Dietary patterns in human populations from Northwest Patagonia during Holocene: an approach using Binford's frames of reference and Bayesian isotope mixing models Archaeological and Anthropological Sciences. DOI 10.1007/ s12520-016-0459-0

Leis, S.A., D. Engle, J. Leslie Jr., J.S. Fehmi \& J. Kretzer. 2015. Comparison of vegetation sampling procedures in a disturbed mixed-grass prairie. Proceedings of the Oklahoma Academy of Science 83: 7-15.

Llano, C. 2009. Photosynthetic pathways, spatial distribution, isotopic ecology, and implications for prehispanic human diets in Central-Western Argentina. International Journal of Osteoarchaeology 19: 130-143.

Llano, C. \& R. Barberena. 2013. Explotación humana de especies vegetales en Patagonia septentrional: el registro arqueobotánico de Cueva Huenul 1 (Provincia de Neuquén, Argentina). Darwiniana N.S. 1 (1): 5-19.
Markgraf, V., C. Whitlock, R. S. Anderson \& A. García. 2008. Late Quaternary vegetation and fire history in the northernmost Nothofagus forest region: Mallín Vaca Lauquen, Neuquén Province, Argentina. Journal of Quaternary Science 24: 248-258.

Mostacedo, B. \& T.S. Fredericksen. 2000. Manual de Métodos Básicos de Muestreo y Análisis en Ecología Vegetal. Editora El País. Santa Cruz, Bolivia.

Movia, C., G.H. Ower \& C.E. Pérez. 1982. Estudio de la vegetación natural de la Provincia del Neuquén, Ministerio de Economía y Hacienda, Secretaría de Recursos Naturales, Neuquén.

Neme, G., A. Gil, C. Otaola \& M. Giardina. 2013. Resource Exploitation and Human Mobility: Trends in the Archaeofaunal and Isotopic Record from Central Western Argentina. International Journal of Osteoarchaeology DOI: 10.1002/ oa.2359.

Páez, M., F. Quintana \& C. Pérez. 2004. Biogeografía de las Regiones Áridas y Semiáridas entre los $35^{\circ}-39^{\circ} \mathrm{S}$, Argentina. Boletín de la Sociedad Argentina de Botánica 39: 171-180.

Rindel, D. 2017 Explorando la variabilidad en el registro zooarqueológico de la provincia del Neuquén: tendencias cronológicas y patrones de uso antrópico. En: El Poblamiento Humano del Norte del Neuquén: Estado Actual del Conocimiento. (Gordón, F., R. Barberena, V. Bernal, eds.), pp. 101-125. Aspha ediciones, Buenos Aires.

Tessone, A., D. Rindel, J.B. Belardi, H.O. Panarello \& R.A. Goñi. 2014. $\delta^{13} \mathrm{C}$ and $\delta^{15} \mathrm{~N}$ Variability in Modern Guanaco (Lama guanicoe) Assemblages in Southern Patagonia: Implications for Zooarchaeological Studies. International Journal of Osteoarchaeology 24: 202-218.

Tieszen, L.L., 1991. Natural variations in the carbon isotope values of plants: implications for archaeology, ecology, and paleoecology. Journal of Archaeological Science. 18, 227-248

Tykot R. 2004. Stable Isotopes and diet: you are what you eat. In Physics Methods in Archaeometry. Proceedings of the International School of Physics "Enrico Fermi", M Martini, M Milazzo, M Piacentini (eds.). Società Italiana di Fisica: Bologna; 433-444 